Research

\title{
Severe acute maternal morbidity in a tertiary care institution
}

\author{
G A Ranatunga ${ }^{1}$, J F Akbar ${ }^{1}$, S Samarathunga ${ }^{1}$, Y A G Perera ${ }^{1}$, L Kariyawasam ${ }^{1}$, J M Kumarasiri ${ }^{1}$
}

Sri Lanka Journal of Obstetrics and Gynaecology 2012; 34: 135-143

\begin{abstract}
Introduction: As the absolute numbers of maternal deaths in an institution are few, maternal death audits may not provide the true picture of service provision. Therefore severe acute maternal morbidity (SAMM), also referred to as near misses, has been proposed as a supplementary indicator for the assessment of the quality of maternity care.
\end{abstract}

Objective: To study SAMM in order to identify strategies which could be adopted to improve quality of care.

Design, Setting and Methods: Using the WHO near-miss criteria, a clinical audit was carried out in all five consultant units of the Castle Street Hospital for Women (CSHW), Colombo from 1st February 2011 to 31st January 2012. Of the admissions to the intensive care, only those who conformed to the WHO near-miss inclusion criteria were studied.

Results: There were 91 cases of SAMM and five maternal deaths out of 16511 live births, giving a maternal mortality ratio of $30.3 / 100,000$ live births, a maternal near-miss ratio of 5.5/1000 live births and a maternal near miss: mortality ratio of 18.2. Of the 91 cases of SAMM, there were $35(38.5 \%)$ cases of major post partum hemorrhage (PPH), 18 (19.8\%) cases of thrombocytopenia and coagulopathy due to Dengue fever, nine $(9.9 \%)$ cases with cardiac dysfunction, five $(5.5 \%)$ cases of liver disease and six (6.6\%) cases of eclampsia. There were 45 cases who had more than one inclusion criterion. Suboptimal management processes identified included incomplete documentation in some cases, non documentation of estimated blood loss in PPH, deficiencies in monitoring in labor and instrumental delivery, non use of uterine tamponade prior to proceeding to hysterectomy, and inadequate knowledge and experience of uterine tamponade insertion.

Conclusions: SAMM is approximately 18 times greater than maternal deaths in the CSHW and

${ }^{1}$ Consultant Obstetricians and Gynaecologists, Castle Street Hospital for Women, Colombo.

Correspondence: G A Ranatunga

E-mail: randa@sltnet.lk approximately $39 \%$ of SAMM is due to major PPH. Dengue fever is an important course of SAMM in the CSHW. Appropriate steps should be adopted to correct the sub optimal practices identified.

Key words: acute maternal morbidity, near-miss

\section{Introduction}

Auditing maternal deaths is an approach commonly used for assessing the quality of care in order to address any identified weakness or failure within the system and take corrective action. In Sri Lanka maternal death was made a notifiable event and clear instructions were provided for the investigation of all maternal deaths from 1989. There have been regular maternal death reviews with proper data collection conducted in Sri Lanka for the past 23 years ${ }^{1}$. Number of maternal deaths has fallen even though it remains somewhat static over the past few years. Maternal death rate for Sri Lanka was 31.1 per 100,000 in $2010^{2}$. When we consider the number of maternal deaths in an institution, the absolute numbers are few to assess the quality of obstetric care given by the institution. As an example the tertiary referral centre Castle Street Hospital for Women (CSHW) in Colombo where 17000 - 19000 deliveries are conducted annually, analyzing $6-8$ maternal deaths will not reflect the true picture of the quality of care given by the Institution. This is further strengthened as some of these maternal deaths are only cases transferred from the periphery to the Intensive Care Unit (ICU) directly, not mothers who had antenatal care at the Institution. Studying cases of women who nearly died but survived a complication during pregnancy, childbirth or postpartum are increasingly recognized as a useful means to examine the quality of obstetric care. These cases are referred to as maternal near misses or severe acute maternal morbidity (SAMM) $)^{3-8}$. It has many similarities and common aspects with those who die of such complications. The World Health Organization (WHO) has standardized definitions for severe morbidity and its main inclusion criteria ${ }^{5,6}$.

\section{Method}

The following WHO near-miss guide inclusion criteria were used ${ }^{6}$. 
Severe maternal complications

* Critical interventions or intensive care unit use

* Life-threatening conditions (near-miss criteria)

- Cardiovascular dysfunction

- Respiratory dysfunction

- Renal dysfunction

- Coagulation/hematological dysfunction

- Hepatic dysfunction

- Neurological dysfunction

- Uterine dysfunction

Slight modifications of these inclusion criteria were made as follows. Transfusion of three or more units of blood (instead of five units) was considered as an inclusion criterion. All cases admitted to ICU were analyzed and the cases who fulfilled the other WHO criteria were included in the study. In addition those who were admitted with cardiac disease and stayed more than 24 hours in the ICU were also included. Detailed information on the cases of PPH (including uterine rupture) and eclampsia were collected using assessment forms adapted from the Scottish study on $\mathrm{SAMM}^{7,8}$. Data collection process was explained to all the medical and nursing staff of the respective units. A lead person from each unit collected the data. Only case record numbers were used to maintain confidentiality. Monthly statistics of ICU admissions were obtained from the indoor morbidity and mortality returns of the hospital and compared and cross checked with data collected from the relevant case notes.

The data were stored confidentially in a computer and analyzed using Microsoft Office Excel 2007 (Microsoft Corporation, USA). The means with 95\% confidence intervals were calculated for the proportions.
The study was approved by the Ethical Review committee of CSHW.

The following Indicators used to monitor quality of obstetric care were calculated ${ }^{5,6}$.

- Maternal near-miss (MNM)

- Maternal death (MD)

- Women with life-threatening conditions $(\mathrm{WLTC}=\mathrm{MNM}+\mathrm{MD})$.

- Severe maternal outcome ratio $(\mathrm{SMOR}=(\mathrm{MNM}$ + MD) per 1000 live births (LB).

- $\mathrm{MNM}$ incidence ratio (MNMIR = MNM/LB).

- Maternal near-miss: mortality ratio (MNM: 1 MD).

- Mortality index $[\mathrm{MI}=\mathrm{MD} /(\mathrm{MNM}+\mathrm{MD})]$.

\section{Results}

The total number of live births for the study period was 16511 . There were and five maternal deaths and were 91 cases SAMM during this period.

Maternal mortality ratio (MMR) was 30.3 per 100,000 live births

Maternal near-miss ratio (MNMR) was 5.5 per 1000 live births

Women with life threatening conditions (WLTC) was 96.

Severe maternal outcome ratio (SMOR) was 5.8 per 1000 live births.

Maternal Near-miss mortality ratio (MNM:1MD) was 18.2 .

Mortality Index (MI) was 5.2\%.

Table 1. Patient Characteristics $(n=91)$

\begin{tabular}{|c|c|c|c|}
\hline Mean age in years & $(95 \% \mathrm{CI})$ & 30.2 & $(29.1-31.2)$ \\
\hline \multicolumn{2}{|l|}{ Range in years } & \multicolumn{2}{|l|}{$18-43$} \\
\hline \multicolumn{2}{|l|}{ Primipara } & 35 & $38.5 \%$ \\
\hline \multicolumn{2}{|l|}{ Parity $2-4$} & 52 & $57.1 \%$ \\
\hline \multicolumn{2}{|l|}{ Parity 5 and above } & 03 & $03.3 \%$ \\
\hline Gestational age in weeks & \multirow[t]{2}{*}{$(95 \% \mathrm{CI})$} & 35.1 & \multirow[t]{2}{*}{$(34.0-36.4)$} \\
\hline Range in weeks & & $9-40$ & \\
\hline \multicolumn{2}{|c|}{ Term pregnancies (More than 37 completed weeks) } & 59 & $64.8 \%$ \\
\hline \multicolumn{2}{|l|}{ Preterm (24 - 37 weeks) } & 26 & $28.6 \%$ \\
\hline \multicolumn{2}{|c|}{ Abortions (less than 24 weeks) } & 06 & $06.6 \%$ \\
\hline
\end{tabular}


Table 2. Study Group according to WHO near-miss criteria ${ }^{5,6} \mathbf{n}=91$

\begin{tabular}{llcl}
\hline Group & \multicolumn{1}{c}{ Near miss criterion } & Total cases & Percentage \\
\hline 1 & Severe post partum haemorrhage & 33 & 36.3 \\
2 & Severe pre-eclampsia & 12 & 13.2 \\
3 & Eclampsia & 06 & 6.6 \\
4 & Sepsis/severe systemic infection & 03 & 3.3 \\
5 & Ruptured uterus & 02 & 2.2 \\
6 & Severe complication of abortion critical interventions & 02 & 2.2 \\
7 & Admission to intensive care unit fulfilling study inclusion criteria & 91 & 100 \\
8 & Interventional radiology & - & - \\
9 & Laparotomy & 22 & 24.8 \\
10 & Use of blood products & & \\
Life-threatening conditions & 40 & 43.9 \\
11 & Cardiovascular dysfunction & 09 & 9.9 \\
12 & Respiratory dysfunction & 01 & 1.1 \\
13 & Renal dysfunction & 02 & 2.2 \\
14 & Coagulation/Haematological dysfunction & 18 & 19.8 \\
15 & Hepatic dysfunction & 05 & 5.5 \\
16 & Neurological dysfunction & 01 & 1.1 \\
\hline
\end{tabular}

Since all the cases were managed in the ICU all 91 cases could be included at least under two WHO near-miss criteria. There were 41 cases who could be included under three and four cases who could be included under more than three criteria.
Of the 91 cases of SAMM, PPH was the leading cause with $35(38.5 \%)$ cases. All $18(19.8 \%)$ cases of coagulation dysfunction had platelet counts less than $50,000 / \mathrm{dl}$ and the five cases of hepatic dysfunction were also due to dengue fever complicating pregnancy.

Table 3. Admissions to intensive care unit according to hospital statistics

\begin{tabular}{lcc}
\hline Indication for admission & Total number & Satisfies the WHO inclusion criteria \\
\hline PPH & 35 & 35 \\
PIH & 109 & 10 \\
Cardiac disease & 39 & 09 \\
HELLP syndrome & 08 & 02 \\
Close monitoring after LSCS & 16 & 0 \\
Close monitoring after NVD & 02 & 0 \\
Upper respiratory tract infection & 01 & 0 \\
Eclampsia & 11 & 06 \\
Dengue fever & 32 & 23 \\
& & {$[18$ (Low platelet count) } \\
Septicaemia & 03 & +5 (Hepatic dysfunction) \\
Renal/liver failure & 02 & 3 \\
Status epilepticus (Neurological dysfunction) & & 2 (Renal failure) \\
Total & 258 & 1 \\
\hline
\end{tabular}

$\mathrm{PPH}=$ Post partum haemorrhage $\mathrm{PIH}=$ Pregnancy induced hypertension $\quad$ HELLP $=$ Haemolysis elevated liver enzymes and low platelets LSCS $=$ Lower segment cesarean section $\quad$ NVD $=$ Normal vaginal delivery 
Although there were 258 cases admitted to the ICU during this period, only 91 fulfilled at least one of the WHO near-miss criteria. In contrast all 91 cases of SAMM had care in the ICU. All PPH cases met the SAMM criteria. Of the 109 women with severe preeclampsia 99 mothers were admitted to the ICU after cesarean delivery for close monitoring and only 10 satisfied the near miss inclusion criteria. Of the 39 women with cardiac disease 30 were admitted to the ICU mainly for intensive monitoring and were discharged from the ICU within 24 hours of delivery. Therefore they were not included in the study. Two cases identified as HELLP syndrome had more than double the value of elevated liver enzymes and low platelets $<60,000 / \mathrm{dl}$. Out of the 11 cases reported as eclampsia only 6 had tonic clonic convulsions, and involuntary movements. Some cases had suspected exaggerated reflexes, and were also treated with magnesium sulphate $\left(\mathrm{Mg} \mathrm{SO}_{4}\right)$ but did not fulfill the WHO inclusion criteria. Therefore they were excluded. A case of status epilepticus mistakenly considered in the eclampsia group was included under the neurological dysfunction criterion. Twenty-three cases of Dengue fever admissions had either abnormal liver profile or platelet count less than $50,000 / \mathrm{dl}$ to be in the SAMM category. One patient with respiratory infection was included in the sepsis group. The patients who were admitted after LSCS and NVD for close monitoring did not conform to the nearmiss inclusion criteria.

Table 4. Cases of post partum haemorrhage

$(\mathrm{N}=35)$

\begin{tabular}{ll}
\hline Mean Age in years $(95 \% \mathrm{CI})$ & $32.2(30.5-33.9)$ \\
Range & $24-43$ years \\
Primiparous & $09(25.7 \%)$ \\
Multiparous & $24(68.6 \%)$ \\
Grandemultiparous & $02(5.7 \%)$ \\
Gestation more than 34 weeks & $32(91.4 \%)$ \\
Gestation less than 34 weeks & $03(8.6 \%)$ \\
\hline
\end{tabular}

Table 5. Mode of delivery and occurrence of post partum haemorrhage $(n=35)$

\begin{tabular}{lcc}
\hline Mode of delivery & No of cases with post partum haemorrhage & Percentage \\
\hline Normal vaginal delivery & 09 & 25.7 \\
Emergency CS & 15 & 42.8 \\
Elective CS & 09 & 25.7 \\
Forceps delivery & 01 & 2.9 \\
Failed forceps leading to emergency CS & 01 & 2.9 \\
Total & 35 &
\end{tabular}

$\mathrm{CS}=$ Cesarean section

Cesarean delivery was associated with $68.5 \%$ of PPH cases. Three cases of emergency CS were carried out in second stage of labour. 
Table 6. Causes of post partum haemorrhage $(n=35)$

\begin{tabular}{lcc}
\hline Cause & Number & Percentage \\
\hline Uterine atony & 20 & 57.1 \\
Placenta previa & 03 & 8.6 \\
Abruption & 02 & 5.7 \\
Uterine inversion & 02 & 5.7 \\
Broad ligament haematoma & 02 & 5.7 \\
Coagulation defect (Thrombocytopenia) & 02 & 5.7 \\
Morbidly adherent placenta & 01 & 2.9 \\
Placenta previa and morbidly adherent placenta & 01 & 2.9 \\
Uterine rupture & 01 & 2.9 \\
Vaginal and cervical laceration & 01 & 2.9 \\
Uterine rupture and cervical laceration & 01 & 2.9 \\
\hline
\end{tabular}

The main cause was uterine atony, identified in 20 (57.1\%). A case of vaginal and cervical laceration and another case of uterine rupture with cervical laceration were due to forceps delivery performed by a middle grade medical officer. There were 04 cases of Placenta previa with one morbidly adherent placenta. Coagulation defect following Dengue Fever with severe thrombocytopenia was associated with two cases of PPH. In four cases more than one factor was associated with $\mathrm{PPH}$.

Table 7. Data well documented in the management of post partum haemorrhage $(n=35)$

\begin{tabular}{lcc}
\hline Procedure & Number & Percentage \\
\hline Assessment of blood loss & 24 & 68.6 \\
Active management of third stage & 32 & 91.4 \\
Use of intravenous infusion of oxytoccin & 30 & 85.7 \\
Intravenous administration of ergometrine & 20 & 57.1 \\
Intravenous administration of tanxaemic acid & 07 & 20.0 \\
Administration of intravenous crystalloids & 25 & 71.4 \\
Active participation of the consultant obstetrician & 18 & 51.4 \\
Adequate information documented in case notes & 15 & 42.9 \\
\hline
\end{tabular}

Blood loss was not documented in 11 (31.4\%) out of 35 cases. Ergometrine was used mainly in the treatment of PPH. Use of tranaxaemic acid was very low. The level of documentation was incomplete in more than half the study group.

Table 8. Management of post partum haemorrhage $(n=35)$

\begin{tabular}{lcc}
\hline Treatment modality & Number & Percentage \\
\hline Condom tamponade & 12 & 34.2 \\
Compression suture & 04 & 11.4 \\
Hysterectomy & 8 (including 05 failed condom tamponade) & 51.4 \\
\hline
\end{tabular}


Condom catheter Tamponade was used in 12 (34.29\%) cases. In five of these cases bleeding continued and hysterectomy was performed. Instilled saline volume was inadequate in two cases. In three patients hysterectomy was performed immediately following the cesarean section by the middle grade operator.

Table 9. The lead personnel involved in surgical management of post partum haemorrhage $(n=35)$

\begin{tabular}{lccc}
\hline $\begin{array}{l}\text { Lead person who performed } \\
\text { surgery/anaesthesia }\end{array}$ & $\begin{array}{c}\text { Hysterectomy } \\
\text { total 18 }\end{array}$ & $\begin{array}{c}\text { Compression sutures } \\
\text { total 04 }\end{array}$ & $\begin{array}{c}\text { Surgical management } \\
\text { total }\end{array}$ \\
\hline Consultant Obstetrician & 13 & 01 & $14(63.6 \%)$ \\
Registrar/ SHO (Obs \& Gyn) & 05 & 03 & $08(36.4 \%)$ \\
Consultant Anaesthetist & 08 & 01 & $09(40.9 \%)$ \\
Anaesthetic Registrar & 10 & 03 & $13(59.1 \%)$ \\
\hline
\end{tabular}

The fact that lessons learnt from these cases were discussed in a subsequent staff meeting was documented only in $11(31.4 \%)$ cases.

\section{Eclampsia $(\mathrm{n}=06)$}

In the six women who had eclampsia the ages ranged from 24-34 with a mean of 30.1 (95\% CI 27.432.7). Five cases of eclampsia occurred in the antenatal period and one was intra partum. Four cases were multiparous mothers. They all had therapeutic as well as prophylactic administration of $\mathrm{Mg} \mathrm{SO}_{4}$ intravenously.

\section{Severe pre-eclampsia $(\mathrm{n}=12)$}

Two cases of HELLP Syndrome were included under this category. Their ages ranged from $23-35$ and the mean age was 28.8 years (95\% CI 25.9 - 30.6). Nine out of 12 patients were primiparous. Labetalol intravenously and orally was the most commonly used antihypertensive while hydralazine intravenously and nifidapine sustained release orally were the other antihypertensives used. Methyl dopa was used only in one patient. In six patients $(50 \%)$ more than one antihypertensive was used. Ten cases were delivered by cesarean section (CS). $\mathrm{Mg} \mathrm{SO}$ was administered before delivery in 08 cases while all mothers had prophylactic $\mathrm{Mg} \mathrm{SO}_{4}$ intravenously after delivery.

\section{Cardiovascular dysfunction $(n=09)$}

There were nine mothers with cardiac disease and they were between 19 - 35 years of age with a mean age 29.2 years (95\% CI 27.6 - 30.8). Of the total six were primigravidae. Seven of them were delivered by CS and two had normal vaginal delivery (NVD).

Table 10. Diagnosed cardiac disease $(n=09)$

\section{Congenital heart disease}

ASD / PHT / R ->L shunt

Residual VSD / PHT

Ebstein anomaly

\section{Acquired heart disease}

Mitral stenosis/PHT

Mitral regurgitation

Mitral valve replacement

\section{Acquired heart disease}

Ischemic heart disease

Concentric left ventricular hypertrophy

Total cases

Mitral valve disease was the commonest (44.4\%). Ischeamic heart disease seen in one patient. 
Table 11. Coagulation/ haematological dysfunction $(n=18)$

\begin{tabular}{lll}
\hline Mean age in years $(95 \% \mathrm{CI})$ & $28.8 \quad(26.0-31.4)$ \\
Range in years & 18 - $43 \mathrm{~s}$ \\
Primiparous & 07 & $(38.9 \%)$ \\
Multiparous & 11 & $(61.1 \%)$ \\
POA <24 weeks & 04 & $(22.2 \%)$ \\
POA 24 - 34 week & 03 & $(16.7 \%)$ \\
POA 34 - 40 weeks & 07 & $(38.9 \%)$ \\
Postpartum & 04 & $(22.2 \%)$ \\
Lowest platelet counts & Range $12000-46000 / \mathrm{dl}$ \\
& Mean $28500 \quad(95 \%$ CI 24250 - 32750) \\
Complication - PPH & $02 \quad(11.1 \%)$ \\
Complication - Gum bleeding/Echymosis & $01 \quad(5.56 \%)$ \\
Needed blood transfusion & $02 \quad(11.1 \%)$ \\
Needed blood products & $03 \quad(16.7 \%)$ \\
Total cases & 18 & \\
\hline
\end{tabular}

All these cases who had platelet count below $50,000 / \mathrm{dl}$ were admitted to ICU with suspected Dengue fever and $61.1 \%$ were multiparous. Mean platelet count was 28500/dls. (95\% CI 24250 - 32750). There were 14 mothers $(88.8 \%)$ of the mothers in the antepartum period and four mothers $(22.2 \%)$ were admitted in the postpartum period. Two patients (11.1\%) needed blood transfusion for management of PPH. Three patients were given blood products including platelets.

\section{Renal dysfunction $(\mathrm{n}=02)$}

Both cases of renal dysfunction were transferred cases from the periphery around 30 - 31 weeks. An 18 year old primigravida had a normal vaginal delivery. Her renal function recovered with haemodialysis and was discharged home with anticoagulation due to venous thrombosis. Second case was a gravida three with no living children had cesarean delivery and had normal urine output with persistent hypertension and abnormal renal function. She was transferred to the renal unit for further investigations.

\section{Septicaemia $(n=03)$}

Two cases had pelvic peritonitis following induced abortion. Both recovered after treatment with intravenous antibiotics and administration of IV fluids, blood and blood products. One, a 30 years old second gravida with one living child, has had an induced abortion around 20 weeks of gestation. The second case was a gravida four with three living children at 11 weeks gestation. The third case had generalized peritonitis with septicemia following elective cesarean section (CS) after two previous cesarean deliveries. She recovered after further surgery and prolonged hospital stay.

\section{Discussion}

The maternal mortality ratio of the hospital was similar to the national rate.

The maternal near-miss incidence ratio (MNMIR) for the institution was 5.5 per 1000 live births. This lies close to the MNMIR of 3.8/1000 deliveries reported from Scotland 8 . MNMR reported from Uganda is $101.7 / 1000$ deliveries 9 . Severe maternal outcome ratio (SMOR) was $5.8 / 1000 \mathrm{LB}$ and is also closer to the Scottish value of 3.9/1000 deliveries compared to the 119/1000 deliveries reported from Uganda. As both these indicators give an estimate of the amount of care and resources that would be needed in an area or facility this probably reflects that reasonably good facilities and resources are available at CSHW.

Maternal near-miss mortality ratio (MNMMR= MNM: 1MD) refers to the ratio between maternal near 
miss cases and maternal deaths. Higher ratios indicate better care. MNMMR for this study was 18.2 while in Scotland it was 49 and in Uganda it was 5.98, .

The higher the mortality index the more women with life-threatening conditions die, indicating low quality of care, whereas the lower the MI the fewer women with life-threatening conditions die indicating better quality of care. The mortality index of CSHW was $5.2 \%$ which is closer to the $2 \%$ reported from Scotland than $14.5 \%$ from Uganda ${ }^{8,9}$. The CSHW is apparently providing good care but there should not be any complacency as there is lot of room for improvement.

From the total deliveries of 16281 there were $0.55 \%$ cases of SAMM. Of the 91 cases of SAMM 51 (56\%) were delivered by CS, 19 (20.9\%) had NVD and three $(3.3 \%)$ had instrumental vaginal delivery (IVD). This accounts for $0.95 \%$ of CS, $0.12 \%$ of NVD and 0. $49 \%$ of IVD.

Three out of the five maternal deaths which occurred during the study period were direct transfers to the ICU. An Increased number of coagulation dysfunction due to Dengue fever $(19.8 \%)$ may have a seasonal variation, but major PPH (38.5\%) and the hypertensive disorders $(19.8 \%)$ appear to be the leading causes. Massive obstetric haemorrhage and hypertensive disorders of pregnancy were the two main morbidity groups in most of the studies reported $^{10-18}$.

As this is the first attempt to study maternal morbidity at CSHW, a few problems were encountered collecting data. Poor documentation was one. All ICU admissions were not considered under the category of SAMM as some of the cases were admitted to ICU only for observation and close monitoring. All cases of SAMM were managed in the ICU for the total duration or for few days at least. However out of 258 admissions to the ICU during the study period the total number who fulfilled the WHO near-miss criteria were only 91 . If the ICU admissions are categorized according to WHO near-miss inclusion criteria, it would facilitate future studies on SAMM.

We observed disparity of the number of morbidity cases in data returns from the wards and the ICU statistics. Some cases where blood loss was not assessed and transfusing less than three units of blood were not included even with surgical interventions like the use of tamponade.

One of the eclampsia cases was directly admitted to the ICU and after delivery she stayed in ICU till she was discharged home. Unfortunately her data was not collected.
If this study is continued with commitment, it should be possible to retrieve accurate data which will help in the final analysis to improve the quality. Detailed data entry in the specific forms as early as the morbidity event will give accurate information. Weekly data collection by a designated officer will be more efficient as extraction of details from the case notes are difficult due to poor documentation. Every effort should be made to improve accurate documentation, especially the visual assessment of blood loss in PPH, administered intravenous fluid, blood, blood products and other medications given.

Majority of the hysterectomies (72.2\%) done for PPH were performed by the Consultant Obstetricians. Consultant Anaesthetist was available for $44.4 \%$ of the cases. Even though middle grade medical officers are well experienced more consultant participation will improve the quality of care and reduce the morbidity. Decision to perform hysterectomy without using less invasive uterine tamponade initially and decision making by middle grade officers was evident in some units. Use of tranexaemic acid and proper use of uterotonics also could be improved by adhering to guidelines. Proper selection of patients for instrumental delivery and correct technique could avoid the complications. Adequate training and refresher courses for middle grade medical officers and all care givers is mandatory.

In two cases of eclampsia proper blood pressure monitoring could have changed the scenario. One was eclampsia in the antenatal period where the change of blood pressure values were not given due attention to plan the management. In the other case of intrapartum eclampsia the blood pressure was not recorded during labour till the convulsions occurred and she was not given any analgesia during labour.

Measures taken after the review of these SAMM cases or lessons learnt from these cases were not indicated in many of the data collection forms. Setting up Risk Management Committees with Risk Management Officers is recommended to establish and improve the risk management culture. Analysis and case presentation of these cases of severe morbidity weekly or every fortnight either in the unit or jointly in the institution (similar to perinatal mortality meetings) will be a progressive step.

Continuation of audit of SAMM will improve clinical practice and reduce preventable morbidity and mortality through the use of current best evidence based practices.

\section{Conclusion}

SAMM is approximately 18 times greater than maternal deaths in the CSHW and approx $38 \%$ of 
SAMM is due to major PPH. Dengue fever was an important cause of SAMM in the CSHW during the study year. Cesarean delivery was associated with higher morbidity. Maternal morbidity during pregnancy and delivery is often preventable. Adhering to National Guidelines and training of care givers, especially the middle grade medical officers, labour room nurses and midwives at regular intervals should be planned. Prevention and proper management of PPH remains a significant strategy in reduction of maternal morbidity. Accurate observations and proper documentation is also an important part of the patient care.

\section{Acknowledgements}

We thank Professor Malik Goonawardane for his thorough review of the manuscript and valuable inputs and Dr. Malith Perera who coordinated the data collection from the wards.

\section{References}

1. Family Health Bureau, Ministry of Health Care and Nutrition of Sri Lanka. Maternal Mortality Decline - The Sri Lankan Experience. Family Health Bureau of Ministry of Health, Nutrition and Welfare. 2003: 47-50.

2. Family Health Bureau, Ministry of Health Care and Nutrition of Sri Lanka. Annual Report on Family Health. National Maternal Mortality Review 2010. Family Health Bureau, Ministry of Health Care and Nutrition of Sri Lanka Colombo 2012.

3. Lale Say, Robert C Pattinson, A Metin Gülmezoglu.WHO systematic review of maternal morbidity and mortality: the prevalence of severe acute maternal morbidity (near miss); Reproductive Health 2004, 1: 3doi:10.1186/ $1742-4755$.

4. Mantel GD, Buchmann E, Rees H, Pattinson RC. Severe acute maternal morbidity: a pilot study of a definition of near- miss. British Journal of Obstetrics and Gynaecology 1998; 105: $985-90$

5. Say L, Souza JP, Pattinson RC. Maternal near miss towards a standard tool for monitoring quality of maternal health care. Best Practice and Research Clinical Obstetrics and Gynaecology 2009; 23: 287-96.

6. The World Heath Organization. Evaluating the quality of care for severe pregnancy complications: the WHO near-miss approach for maternal health. WHO guide, The World Health Organization, Geneva, 2011.

7. Scottish Confidential Audit of Severe Maternal Morbidity 5th Annual Report 2007. The Scottish Executive Health Department. Reproductive Health Programme, Healthcare Improvement Scotland. 2009.

8. Brace V, Penney G, Hall M. Quantifying severe maternal morbidity: a Scottish population study. British Journal of Obstetrics and Gynaecology 2004; 111: 481-4.

9. Kaye D, Mirembe F, Aziga F, Namulema B. Maternal mortality and associated near-misses among emergency intrapartum obstetric referrals in Mulago Hospital, Kampala, Uganda. East African Medical Journal 2003, 80: 144-49.

10. Filippi V, Alihonou E, Mukantaganda S, Graham WJ, Ronsmans C. Near misses: Maternal morbidity and mortality. Lancet 1998; 351: 145-6.

11. Wagaarachchi PT, Graham WJ, Penney GC,McCaw-Binns A, Yeboah Antwi K, Hall MH. Holding up a mirror: changing obstetric practice through criterion-based clinical audit in developing countries. International Journal of Gynaecology and Obstetrics 2001; 74(2): 119-31.

12. Sivalingam N, Looi K, Clinical Experience with Management of 'Near-miss' Cases in Obstetrics. Medical Journal of Malaysia 1999, 54: 496-503.

13. Waterstone M, Bewley S, Wolf C. Incidence and Predictors of Severe Obstetric morbidity: Case control study. British Medical Journal 2001, 322: 1089-94.

14. Hall MH. Near misses and severe maternal morbidity. In Why mothers die 1997-1999. The confidential enquiries into maternal deaths in the United Kingdom. London: RCOG Press: Department of Health, Welsh Office, Scottish Home and Health Department, Department of Health and Social Sciences, Northern Ireland; 2001: 323-5.

15. Baskett TF, Sternadel J. Maternal intensive care and nearmiss mortality in obstetrics. British Journal of Obstetetrics and Gynaecology 1998; 105: 981-4.

16. Alsayali ARA, Baloul AMA. Emergency obstetric hysterectomy: 8-year review at Taif Maternity Hospital, Saudi Arabia. Annals of Saudi Medicine 2000; 20: 454-6.

17. Nasrat HA, Youssef MH, Marzoogi A, Talab F. 'Near miss' obstetric morbidity in an inner city hospital in Saudi Arabia. Eastern Mediterranean Health Journal 1999; 5: 717-26.

18. Waterstone M, Bewely S,Wolf C. Incidence and predictors of severe morbidity: case-control study. British Medical Journal 2001; 322:1089-94. 